\title{
Multi-Point Single-Antenna Sensing Enabled by Wireless Nested Split-Ring Resonator Sensors
}

\author{
Burak Ozbey, Member, IEEE, Vakur B. Ertürk, Member, IEEE, Ozgur Kurc, \\ Ayhan Altintas, Senior Member, IEEE, and Hilmi Volkan Demir, Senior Member, IEEE
}

\begin{abstract}
In this paper, simultaneous multi-point wireless sensing is proposed and demonstrated via multiple sensors in nested split-ring resonator (NSRR) geometry coupled to a single illuminator antenna. In this passive multi-point sensing system, each probe in the sensor array is assigned a nonoverlapping spectral interval for frequency shift in response to local mechanical loading around a unique operating resonance frequency in the band of the antenna. Here, it is shown that the antenna is capable of capturing the responses from all probes in a single frequency sweep. Furthermore, the intercoupling between the array elements and the effect of antenna illumination on the coupling are experimentally investigated in a systematic way. In addition, as a proof-of-concept real-life application in structural health monitoring, two NSRR sensors are located inside a concrete beam to monitor the strain forming on reinforcing bars, and this dual-probe system is demonstrated to record strain simultaneously via both of the embedded probes.
\end{abstract}

Index Terms-Wireless passive sensor, displacement sensor, strain sensor, nested split ring resonator (NSRR), multi-point sensing, multiple sensors, structural health monitoring (SHM).

\section{INTRODUCTION}

$\mathbf{M}$ ETAMATERIAL-BASED sensors have come into prominence in recent years as a result of the advanced capabilities and advantages they introduced in terms of the sensitivity and the resolution levels of the sensed quantity and compact footprints in comparison to traditional structures [1]. Several metamaterial-based structures including variants of split ring resonators (SRRs) and composite right-/left-handed transmission lines (CRLH TLs) have been employed in a variety of applications including the sensing of permittivity of

Manuscript received July 26, 2016; accepted August 22, 2016. Date of publication August 29, 2016; date of current version September 28, 2016. The work of H. V. Demir was supported by the European Science Foundation and Turkish National Academy of Sciences. The associate editor coordinating the review of this paper and approving it for publication was Prof. Tarikul Islam.

B. Ozbey and V. B. Ertürk are with the Department of Electrical and Electronics Engineering, Bilkent University, Ankara TR-06800, Turkey (e-mail: ozbey@ee.bilkent.edu.tr; vakur@ee.bilkent.edu.tr).

O. Kurc is with the Department of Civil Engineering, Middle East Technical University, Ankara TR-06800, Turkey (e-mail: kurc@metu.edu.tr).

A. Altintas is with the Communications and Spectrum Management Research Center (ISYAM), Department of Electrical and Electronics Engineering, Bilkent University, Ankara TR-06800, Turkey (e-mail: altintas@ee.bilkent.edu.tr).

H. V. Demir is with the Department of Electrical and Electronics Engineering and the Department of Physics, Institute of Materials Science and Nanotechnology (UNAM), Bilkent University, Ankara TR-06800, Turkey, and also with the School of Electrical and Electronic Engineering and the School of Physical and Mathematical Sciences, Nanyang Technological University, Singapore 639798, Singapore (e-mail: volkan@stanfordalumni.org).

Digital Object Identifier 10.1109/JSEN.2016.2604020 dielectric materials [2], biological molecules [3], displacement and strain [4], [5], temperature [6], rotation [7], as well as refractive index in terahertz [8] and optical frequencies [9], some of which use plasmonic resonances [10]. SRR-based structures offer a cheap and efficient way of passive and semipassive (e.g. RFID) sensing in contrast to electronic circuitry that constantly requires powering up. On the other hand, majority of the SRR-based sensors reported in literature have been exploited in wired form, usually in the form of 2-port devices. In real-life applications, however, wireless sensors are much more practical and are increasingly in high demand.

Another important requirement about sensors is the 2-D or 3 -D sensing capabilities in array configuration or via several resonances corresponding to different features of a single structure. Such a network of sensors can provide valuable information for the characterization of the planar and spatial distribution of the sensed quantity. In this regard, wired SRR-based sensors have been previously shown: In [11], Withayachumnankul et al. utilized the coupling of different SRR structures to a microstrip transmission line for thin-film sensing. Each structure created a resonance at a specific frequency and a shift observed in each dip provided information related to the dielectric properties as well as the location of the sample, increasing the throughput. In [12], a similar approach was used by Puentes et al. for resolving the relative changes in 2-D dielectric properties of organic tissues by a 12-element SRR array. In [13], Horestani et al. demonstrated an alignment and displacement sensor based on two movable broadside coupled SRRs oriented at $90^{\circ}$ angle with respect to each other to detect changes in $x$ and $y$ directions. Although these sensors provide better comprehension and characterization of the planar distribution of their respective sensed quantities, they are all arranged in wired configuration and the samples have to be placed directly in contact with the sensors. This constitutes a major problem preventing their use in important applications, e.g., in harsh environmental conditions, remote sensing platforms, and in structural health monitoring (SHM) when the sensors have to be embedded inside concrete. A passive wireless sensor was shown by $\mathrm{Xu}$ and Huang for detection of the location and propagation of cracks in a material, on which microstip patch antennas of different resonant frequencies are located [14]. When a crack is observed at a point where the antenna is placed, the antenna radiation characteristics become subjected to a change and this can be observed by sending and recovering the backscattered signal by an interrogator antenna. By this technique, combined with spatial division, where two antennas 
with the same resonant frequency are switched ON and OFF, specific response from four different antenna-sensors can be obtained. This frequency division technique can be improved to a wireless passive 2-D sensing mechanism by employing multiple sensors with a predefined resonant frequency and bandwidth, where the responses from every sensors can be captured in a single frequency sweep.

In this work, wireless 2-D sensing is proposed and demonstrated as a proof-of-concept using comb-like nested SRR (NSRR) probes in a real life application of sensing the displacement and strain forming on reinforcing bars which are embedded in a concrete beam. Comb-like NSRR geometry was first proposed by our group in [15] for an enhanced sensitivity compared to traditional SRRs, and was used as a strain [15] and displacement [16], [17] sensor for biomedical and SHM applications, respectively. Works that incorporate other passive wireless structures for displacement and strain have previously been shown mainly for RFID-based sensing [18]-[23]; however, the sensors were not exploited in a configuration enabling a multi-point sensing by a single antenna. Here, it is shown for the first time that several NSRR probes can be located in a 2-D configuration while different wireless readouts can be acquired concurrently from each probe. This is possible by assigning each probe in the sensor array to a different range of operating frequency through modification of their initial separation distances and/or jumper lengths [24]. In this configuration, the bandwidth allocated for each probe determines the number of array elements as well as the dynamic range of the system while the illumination pattern of the antenna is effective in the strength of the coupling. In such an array, a point of consideration is the effect of inter-coupling, which sets the coupling between the array elements. These effects are systematically studied and approaches of sensing enhancement are discussed in the paper.

The organization of the paper is as follows: In Section II, the proposed multi-point sensing scheme is described and demonstrated through experiments in which two different methods are introduced for spectral separation of the sensor resonances. In this section, the effects of the antenna illumination on coupling are also discussed. In addition, the undesired effect of inter-coupling among the sensors in the array is explored via experiments. In Section III, an important application of the proposed sensing scheme in SHM is demonstrated for the case when two different NSRR probes are utilized in a reinforced concrete beam and the antenna outside the beam is used to detect the strain and displacement values measured from each of the sensors. Section IV concludes the paper.

\section{Multi-Point Sensing}

\section{A. Theory}

The main idea behind this work is to generate a coupling between the antenna and each of the NSRR probes in the array. The antenna illuminates the array elements and also collects the scattered waves. Each NSRR probe is assigned a specific resonance frequency $\left(f_{\text {res }}\right)$ and a frequency interval $(\Delta f)$. Through these parameters, an electromagnetic channel is created between the antenna and each NSRR probe. Since these parameters are set in such a way that there are no intended

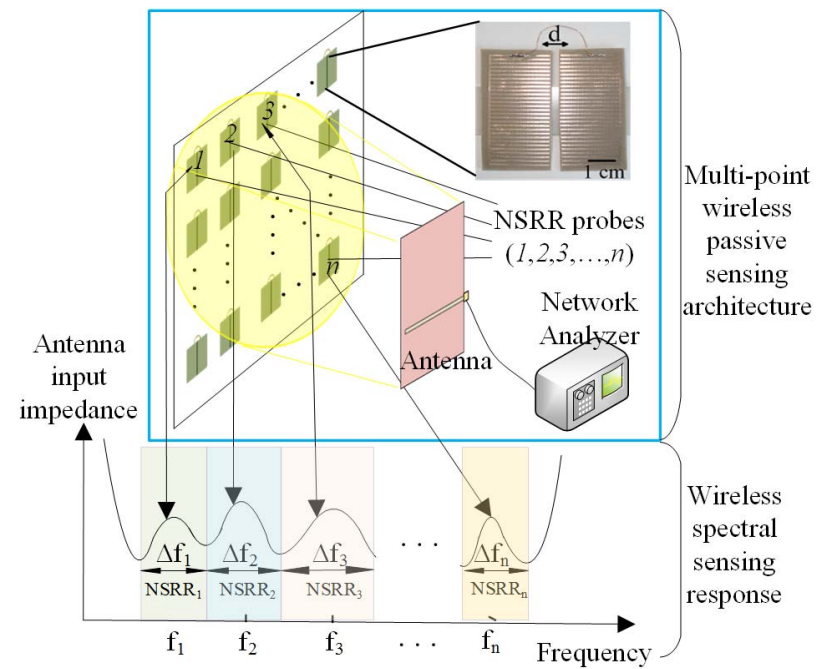

Fig. 1. Concept of the proposed multi-point wireless passive architecture consisting of $n$ nested split ring resonator (NSRR) probes and wireless spectral sensing response of each sensor shown on the frequency-antenna input impedance characteristics.

spectral overlaps, a multi-point system is formed using only a single antenna where the antenna can acquire information from each sensor simultaneously in a single frequency sweep of the network analyzer (VNA). A conceptual diagram summarizing the working principle of the sensing system is illustrated in Fig. 1.

The response of each NSRR probe can directly be observed at the input impedance of the antenna due to the coupling created by the array elements and the antenna. The quality of coupling is directly related with how well the antenna illuminates the NSRR probes. This means that the antenna should electromagnetically "see" each NSRR probe in the array to capture their respective responses. Once an NSRR probe is electromagnetically in the sight of the antenna, it manifests itself as a local peak in the input impedance of the antenna (or the reflection coefficient of the coupled system) at the resonance frequency of that specific NSRR probe. The frequency of this peak shifts as the sensed quantity is subject to a change. This is due to the capacitance change at the NSRR probe. For instance, in order to detect the relative displacement change along two points by a passive and wireless method, a variant of the comb-like NSRR probe can be utilized [16], [17], [24]. In this structure, the comb-like NSRR probe is split symmetrically into two parts that are electrically shorted by a thin jumper to form the normally continuous uppermost ring. These two freely movable comb-like NSRR parts are point-attached to a surface or a structure along which the displacement is to be measured. When the distance between these two points changes, the capacitance of the overall probe also changes (decreasing while the gap between the points increases and vice versa). This way, the comb-like NSRR probe, which by itself is a 1-D strain sensor, is transformed into a displacement sensor. The resonance frequency of the structure is naturally related to the number of the split rings, but it is also determined by the length of the jumper. This is because the jumper introduces a significant level of additional 


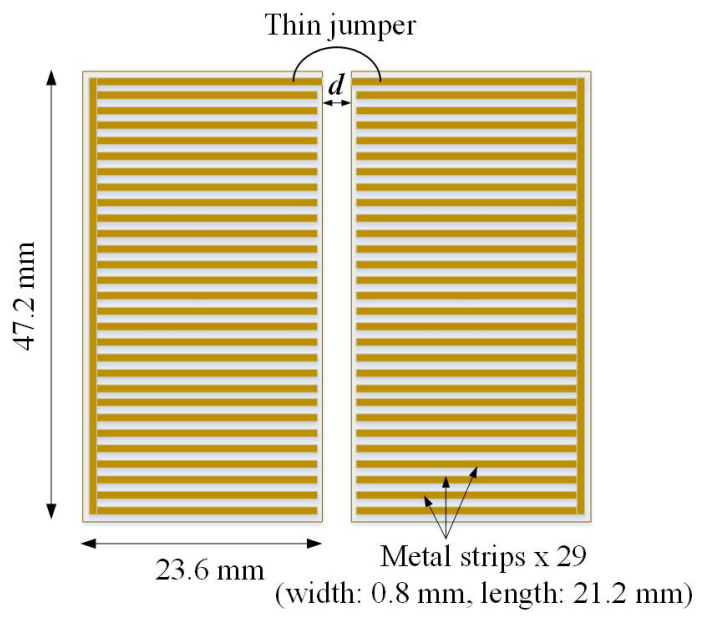

Fig. 2. The modified nested split-ring resonator structure used in this work.

inductance to the NSRR probe. Since both the capacitance and the inductance are effective in setting the resonance frequency of the structure, a change in the distance between the two attachment points creates a shift at the peak of the overall system response. The modified comb-like NSRR structure is shown in Fig. 2 and the inset of Fig. 1. The dimensions of the design used in this work are also displayed in Fig. 2. Further detailed information regarding the standard and modified comb-like NSRR probe structures can be found in [15]-[17] and [24].

The term "resonance" is used for the tracked local peak frequency observed from the antenna reflection spectrum. The reason for this can be described as follows: The behavior of the NSRR probe when it is decoupled from the antenna can be identified by placing the NSRR inside a waveguide and inspecting the reflection coefficient. In such an experiment, the resonance is in the form of a dip in the reflection plot (much of the energy sent to the probe is stored in the probe, and does not return back). However, in the coupled case, the NSRR probe stays within the near-field of the antenna, and the valley behavior is not observed. Instead, a peak that shifts is observed in the antenna reflection coefficient spectrum as the displacement $d$ is varied. This shift corresponds to a shift of the valley observed in the waveguide experiment (when only the NSRR response is present) when the displacement between the NSRR probe parts is changed. Since both shifts are characterized to yield the same behavior in the coupled and the decoupled cases, the peak is defined as resonance.

\section{B. Verification via Experiments}

In order to demonstrate the simultaneous multi-point 2-D sensing, experiments were carried out. The setup for an experiment, where the number of array elements $(n)$ is 3, is given as an inset in Fig. 3. In the experiments, an array of modified comb-like NSRR probes was illuminated via a microstrip single-slot antenna (see Fig. 3 inset). For separation of the frequency intervals corresponding to each sensor, the modification of initial edge-to-edge distances ( $d$ as shown in Fig. 3) between the two parts of each probe in
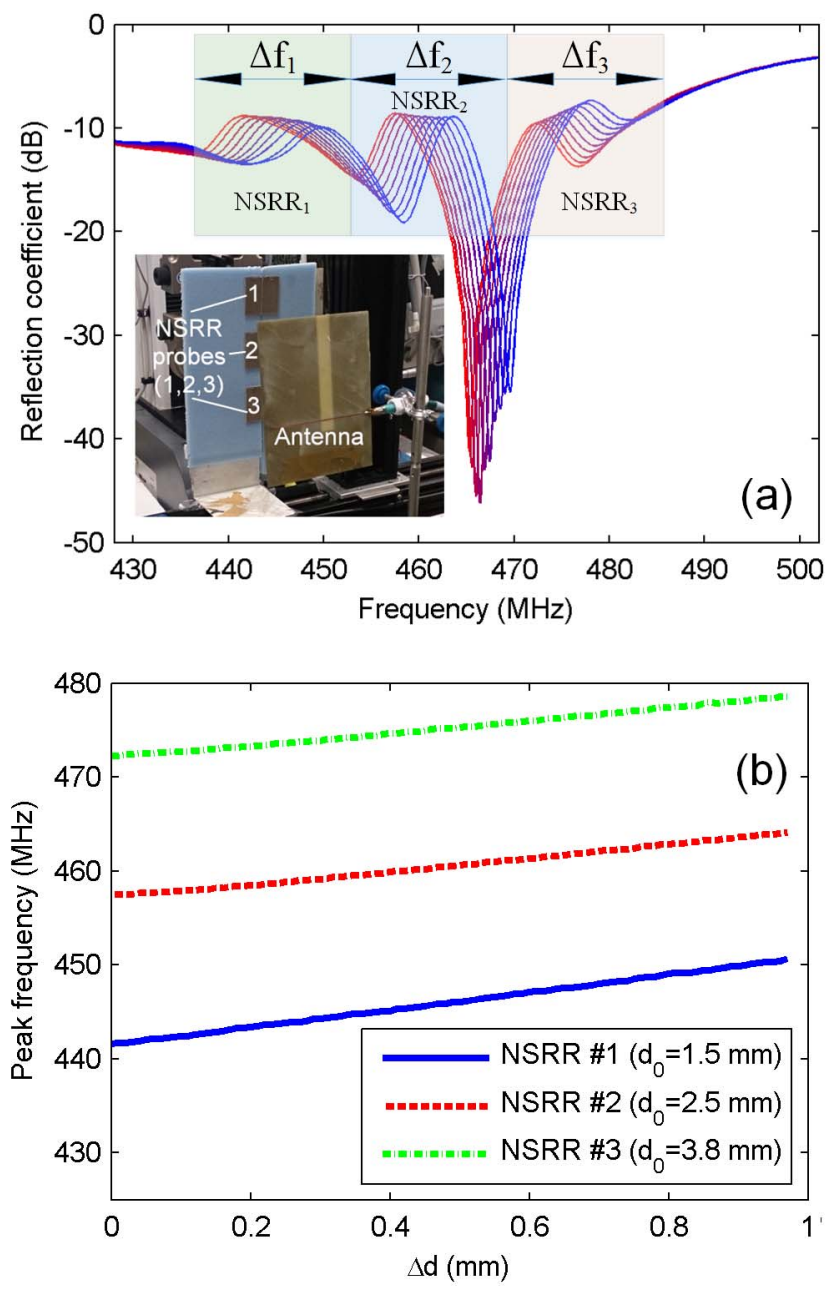

Fig. 3. (a) The shift of resonance frequency for each NSRR probe in the sensor array $(n=3)$ when $d$ is increased from $d_{0}$ to $d_{0}+1 \mathrm{~mm}$. The resonance frequencies of each probe are determined by modification of $d_{0}$. Left (inset): Experiment setup. (b) The change of the resonance frequency versus the change in $d$ for each probe.

the array was used. Each sensor incorporated a $0.1 \mathrm{~mm}$ diameter jumper with a specific length $(l)$, which was selected exactly as $3.5 \mathrm{~cm}$ for all probes. Three local frequency peaks corresponding to each NSRR probe can be observed at the reflection coefficient of the antenna due to coupling, as shown in Fig. 3. Although each of the three NSRR probes is clearly distinguished in the form of a peak, the coupling strengths are observed to be different for each resonance. As explained, this is a result of the variation in antenna illumination. The probes that are positioned at the edges with respect to the antenna are relatively less well-illuminated, leading to worse distinguishability. The resonances which are closer to the limits of the antenna bandwidth may also have poorer coupling strengths. On the other hand, once an NSRR-probe is seen by the antenna, it is also possible to track the shift of that peak which makes the sensing possible. Here, $d$ is increased from specific starting points $\left(d_{0,1}=1.5 \mathrm{~mm}, d_{0,2}=2.5 \mathrm{~mm}\right.$, $d_{0,3}=3.8 \mathrm{~mm}$ ) by $1 \mathrm{~mm}$ via a controlled displacement setup. This results in a shift of approximately $8 \mathrm{MHz}, 6 \mathrm{MHz}$ and $6 \mathrm{MHz}$ in the resonance frequency of the first, second 

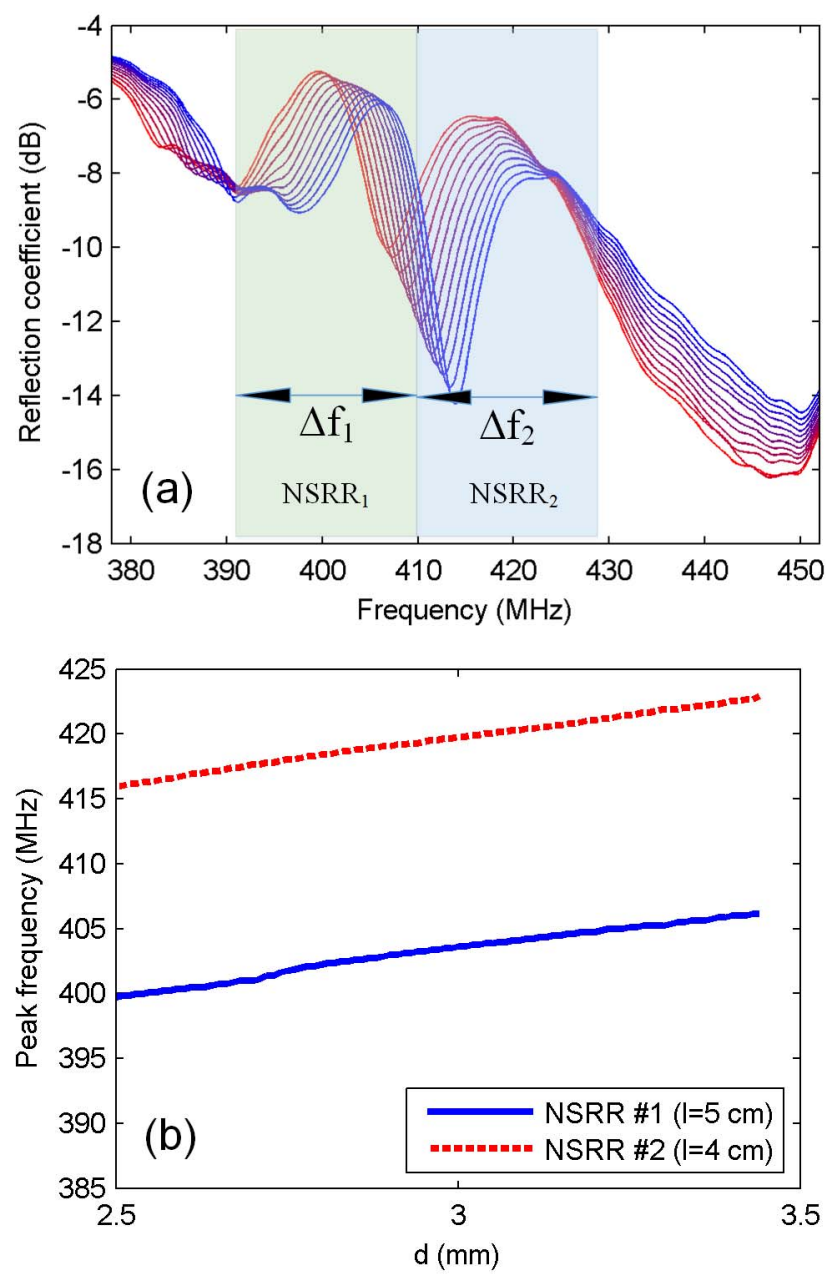

Fig. 4. (a) The shift of resonance frequency for each NSRR probe in the sensor array $(n=2)$ when $d$ is increased from 2.5 to $3.5 \mathrm{~mm}$. The resonance frequencies of each probe are determined by modification of $l$, where $l_{1}=5 \mathrm{~cm}$ and $l_{2}=4 \mathrm{~cm}$. (b) The change of the resonance frequency versus the change in $d$ for each probe.

and third peaks, respectively. The frequency shift versus the change in $d$ (denoted as $\Delta d$ ) is shown in Fig. 3-b. In order to transform the sensing into measurement, all the sensors in the array configuration has to be characterized separately in terms of the respective change of their resonance frequencies with $d$. This is also required for determining the sensitivity of each sensor, which can be defined as the ratio of the frequency shift to the change in $d$.

As mentioned earlier, the separation of the resonance frequencies for the same NSRR geometry can be implemented by either assigning each sensor a different starting $d$, or a different jumper length, $l$. The experiment result showing the multipoint sensing by two NSRR probes having the jumper lengths of $4 \mathrm{~cm}$ and $5 \mathrm{~cm}$ is presented in Fig. 4-a and the frequency shifts versus $d$ are shown in Fig. 4-b. Here, $d_{0}$ is $2.5 \mathrm{~cm}$ and the same for both NSRR probes, while the modification of $l$ by $1 \mathrm{~cm}$ is observed to shift the resonance frequency by around $15 \mathrm{MHz}$. Either of these two methods can be utilized for determining the resonance frequencies and the frequency shift intervals.

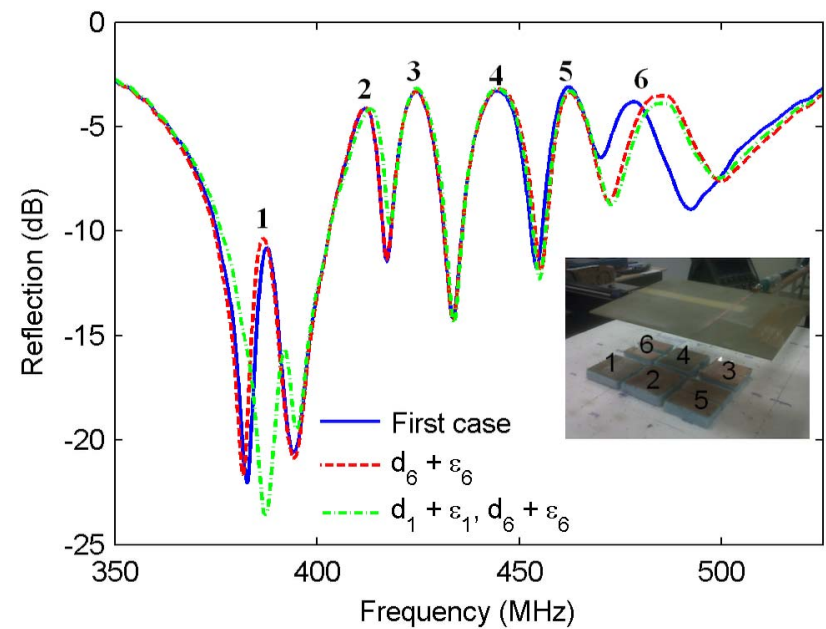

Fig. 5. The result of an experiment where $n=6$ : Initial case is shown in blue, the case where $d$ is slightly increased for NSRR \# 6 is shown in red, and the case where $d$ is slightly increased for NSRR \# 1 is shown in green (for this case, $d$ remains the same as the second case. All other $d$ 's remain the same. The experiment setup is shown in the right inset. Simulation results of a $4 \times 4$ array $(n=16)$ with $d$ varying between 1 to $4 \mathrm{~mm}$ in $0.2 \mathrm{~mm}$ steps, and when $d$ is increased by $100 \mu \mathrm{m}$.

The idea proposed in this work depends on allocating a pre-defined $f_{\text {res }}$ and a $\Delta f$ for each sensor. Along with the antenna bandwidth $(B W), \Delta f$ is the factor that determines the dynamic range of each sensor and the number of sensors in the array. A theoretical limit on the maximum number of array elements $n$ is then $n=\frac{B W}{\Delta f}$. An experiment result where $n$ is 6 is shown in Fig. 5. In this experiment, the NSRR sensors, whose frequencies are set by either modification of $d$ or $l$, make their appearance as peaks, resulting in 6 different peaks at corresponding frequencies. The initial case is given in Fig. 5 (blue curve). Then, $d$ of NSRR \# 6 is increased slightly while other NSRR sensors remain untouched, causing the peak at the highest resonance frequency to shift right (red curve). Finally, $d$ of NSRR \# 1 is increased slightly while it remains the same for all other sensors (see the green curve). Here, the critical point is the following: $d$ of the NSRR \# 6 is not returned back to its original position shown in the blue curve, but instead, it remains in the position shown in the red curve where its $d$ had slightly been increased. Therefore, in order to see the effect of changing $d$ of NSRR \# 1, the red and the green curves should be compared. This experiment shows that it is possible to apply multi-point sensing technique to an array where $n=6$. Even higher number of sensors are also theoretically feasible. The limits of the system are tested with a full-wave simulation in CST Microwave Studio, where $n=16$ and each sensor is assigned a $d$ from 1 to $4 \mathrm{~mm}$ with $0.2 \mathrm{~mm}$ steps. In the simulations, the sensors are illuminated by a plane wave. The resulting peaks in the antenna reflection coefficient for the initial case are shown in Fig. 6 (blue curve). Then, $d$ is increased by $100 \mu \mathrm{m}$ at every sensor, resulting in a frequency shift, which can also be observed in Fig. 6 (red curve). Both the experiment result where $n=6$ and the simulation result of a $4 \times 4$ array show that the multi-point sensing is applicable to higher number of sensors. 


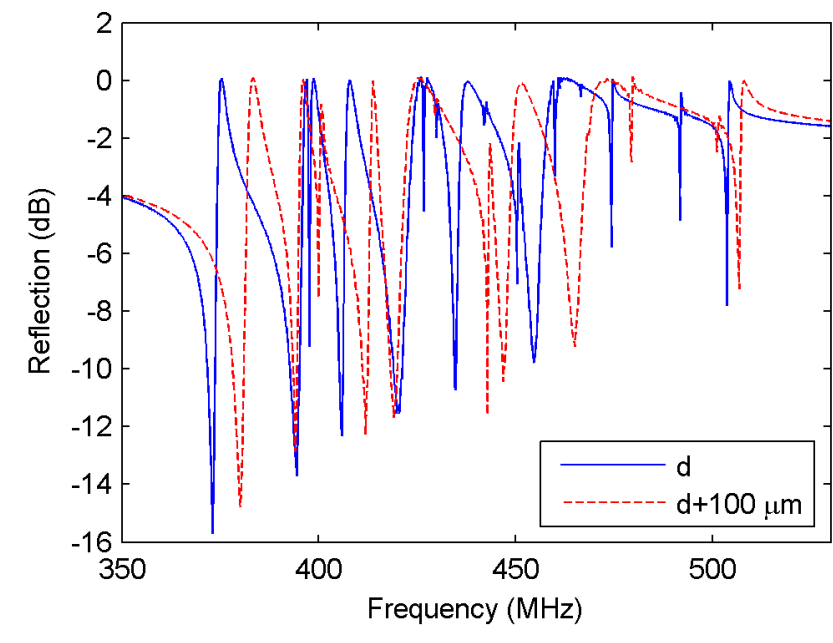

Fig. 6. Simulation results of a $4 \times 4$ array $(n=16)$ with $d$ varying between 1 to $4 \mathrm{~mm}$ in $0.2 \mathrm{~mm}$ steps, and when $d$ is increased for each NSRR probe in the array by $100 \mu \mathrm{m}$.

In the measurements, VNA is important in determining the minimum step size (of displacement or strain), which we call the resolution. The measurement limit of the resolution can be deduced from the sensitivity of the system, which is defined as the measured frequency shift over a displacement range as follows: For example, if the minimum distinguishable frequency that can be measured by the VNA is $1 \mathrm{kHz}$, and the average sensitivity of the system in the $1-3 \mathrm{~mm}$ range is $12.7 \mathrm{MHz} / \mathrm{mm}$ as shown in [16] (where the $d-f$ curve is highly linear), then the resolution limit is found as $(1 \mathrm{kHz}) /$ $(12.7 \mathrm{MHz} / \mathrm{mm})=78.7 \mathrm{~nm}$. This, of course, is a theoretical limit, and it is known that the resolution of the sensing system is 0.5 to $1 \mu \mathrm{m}$ based on our initial experiments presented in [16] and [24]. If we wish to have a measurement range of $1 \mathrm{~mm}$ for each sensor in the array, then, assuming a $100 \mathrm{MHz}$ antenna bandwidth, the maximum number of array elements is found as $100 \mathrm{MHz} / 12.7 \mathrm{MHz} / \mathrm{mm} \approx 8$. This discussion leads to the conclusion that the main compromise of the system is between its spatial and spectral capacities. In order to have a higher resolution of the sensed quantity, one has to locate as many sensors as possible over the area to increase the spatial capacity, e.g. for determining the 2-D strain forming on the surface of a material. However, division of the available bandwidth to many intervals implies a narrow $\Delta f$ for each sensor, which in essence determines the dynamic range of the system, or, the difference between the minimum and maximum strain values measurable by the system. Similarly, to increase the dynamic range, one has to give away from the resolution, which is determined by the number of sensors in the system.

Apart from this trade-off, a point that needs to be improved in the system is $D_{m}$, which is the monitoring distance (distance between the sensors and the antenna), since until now, the proposed wireless sensing scheme has only been limited to the near-field of the antenna, which ensures a distance of a few tens of centimeters for the selected frequency range. This distance is proportional to the operating wavelength and is even lower for higher frequencies. However, an interesting configuration of the wireless multi-point sensing system is to employ sensors assigned to exactly the same frequency and to keep them close to each other at a distance with higher inter-coupling. Since it is known that increasing the monitoring distance $\left(D_{m}\right)$ decreases the depth of the notch (or the coupling strength of the resonance) [16], the superposed responses from multiple sensors tuned to the same resonance frequency can be used to increase the distance between the antenna and the NSRR probes. This is enabled by a deeper notch due to the superimposed responses. In the experiments, it was observed that the placement of two NSRR probes tuned to the same frequency increased the coupling strength approximately by $1 \mathrm{~dB}$.

\section{The Effect of Illumination on Coupling Strength}

It was previously mentioned that the illumination of the sensors determines the coupling strength between the antenna and the NSRR probe. The coupling strength, which is closely related with the quality of the sensing, can be understood from the depth of the notch in the antenna reflection coefficient created due to the NSRR probe. In order to understand the two dimensional effects of the antenna illumination pattern on the coupling between the NSRR probe and the antenna, the following experiment is performed: An NSRR probe is positioned at different times in front of the antenna at 320 different points in an area which is $20 \mathrm{~cm} \times 38 \mathrm{~cm}$ wide (see Fig. 7-a). At each point, $\left|S_{11}\right|$ is measured and the depth of the notch is found after subtracting from each curve the $\left|S_{11}\right|$ for the case when there is no NSRR probe present. For the experiment, $D_{m}$, the distance between the antenna and the NSRR probe, is $10 \mathrm{~cm}$, while $d=0 \mathrm{~mm}$ and $l=4 \mathrm{~cm}$. The color map (in $\mathrm{dB}$ ) of the resonance depths obtained with this experiment is shown in Fig. 7-b. The sketch showing the antenna position is also superimposed on the coupling strength pattern in yellow color. As can be observed in the figure, the coupling is directly related with the illumination pattern of the antenna. The microstip single-slot antenna is known to radiate from the slot introduced at the other side of the feedline. The strongest coupling is observed at the point where the feedline meets the slot (around the center of the antenna), diminishing gradually by forming elliptic contours due to the slot. This coupling strength pattern demonstrates the interaction between the sensing system elements in two dimensions, where the third dimension which is normal to this plane determines $D_{m}$.

\section{Inter-Coupling Between Sensors}

A critical point regarding the array structures is the intercoupling, which is the interaction between the array elements. This leads to a change in the total capacitance of the coupled system which in turn creates a shift of resonance frequency, adversely affecting the sensing characteristics described above. This inter-coupling, which is more dominant when the array elements get closer to each other, was characterized by experiments. In these experiments, an NSRR probe was placed at a fixed position just across the point of the antenna where the feedline and the slot of the antenna meet (the antenna was also kept stationary at a distance of $10 \mathrm{~cm}$ ) while another probe was located at several changing positions along the 


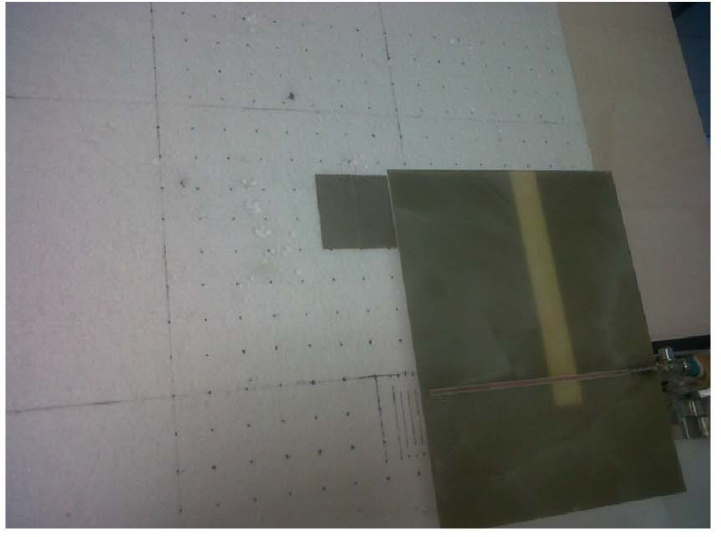

(a)

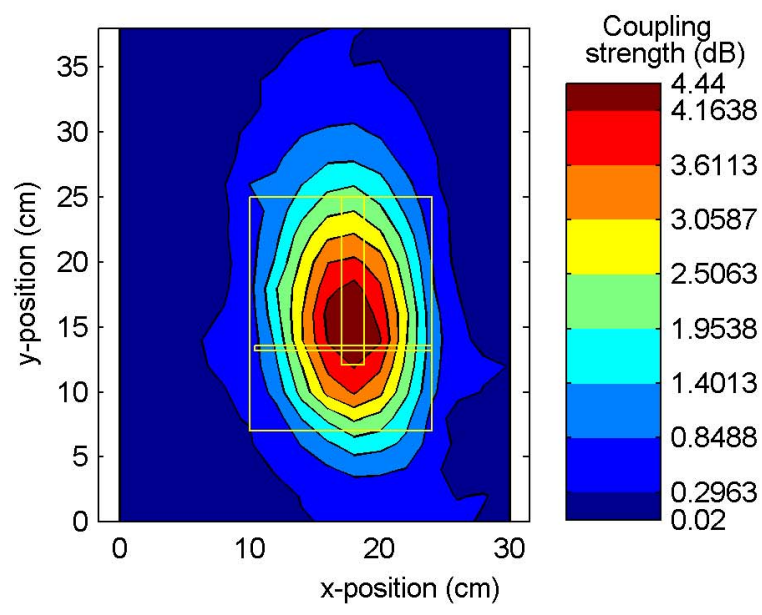

(b)

Fig. 7. (a) The points on which the NSRR probe is positioned for the coupling pattern experiment. (b) Color map demonstrating the coupling strength between the NSRR probe and the antenna in a 2-D pattern. The antenna position is also shown on the plot.

left, right, bottom and top axes of the fixed probe. The edge-toedge distances $(D)$ between the stationary and moving probes were changed from 0 to $40 \mathrm{~mm}$ in four directions (upwards, downwards, rightwards and leftwards as shown in Fig. 8-a).

The diagonal variation was skipped since it was observed that the diagonal placement of the NSRR probes with respect to each other has negligible impact on the coupled system resonance frequency. In this experimental procedure, the intercoupling was identified by tracking the variation of the fixed NSRR probe resonance frequency due to the placement of the second NSRR probe in the vicinity of the fixed one. The experiment setup is shown in Fig. 8-a. The absolute fractional change of fixed NSRR resonance frequency is shown in Fig. 8-b and Fig. 8-c for two different experiments, respectively. In the first experiment, the resonance frequencies of the two probes are set by changing the jumper length: The fixed NSRR probe has an $l$ of $4 \mathrm{~cm}$ (corresponding to $f_{\text {res }}=405 \mathrm{MHz}$ ) and the NSRR probe which is moved along the top, bottom, right and left axes of the fixed probe, has an $l$ of $8 \mathrm{~cm}$. For both probes, $d=0$. As for the second experiment, the resonance frequencies are set by changing the
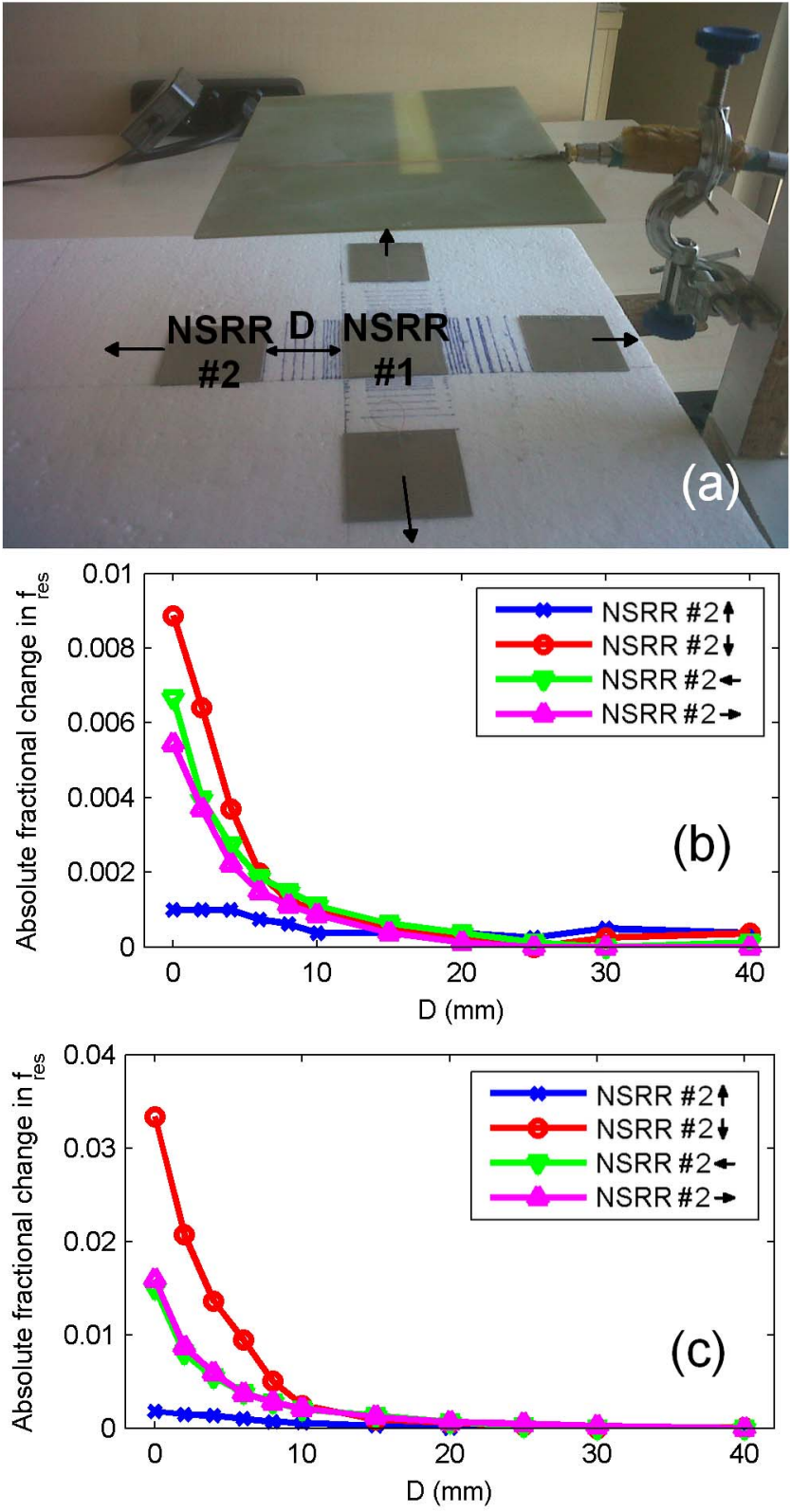

Fig. 8. Experiments related to the inter-coupling in the sensing system where $n=2$ : (a) Experiment setup. (b) Fractional variation in the resonance frequency of an NSRR probe $\left(l_{1}=4 \mathrm{~cm}\right)$ when another probe $\left(l_{2}=8 \mathrm{~cm}\right)$ is located at edge-to-edge distances $(D)$ varying from 0 to $40 \mathrm{~mm}$ along top, bottom, right and left axes. $d=0$ for both probes. (c) Same experiment except the resonance frequencies are set by assigning the probes different $d_{0}$ 's instead of different $l$ 's. $d_{1}=2 \mathrm{~mm}, d_{2}=0$, and $l=3.5 \mathrm{~mm}$ for both cases.

initial edge-to-edge distances: The fixed probe has a $d_{0}$ of $2 \mathrm{~mm}$ (corresponding to $f_{\text {res }}=450 \mathrm{MHz}$ ) while the moving probe has a $d_{0}$ of 0 . For both probes, $l=3.5 \mathrm{~cm}$. As expected, for both cases, the inter-coupling is observed as diminishing as the distance between the two NSRR probes is increased. In the worst case scenario of placing the two probes in a touching position, the maximum deviation from the standard resonance frequency is exhibited for the case when the moving probe is placed at the bottom of the stationary probe.

The inter-coupling experiment is useful in the characterization of the effect of the position of the sensors on each 

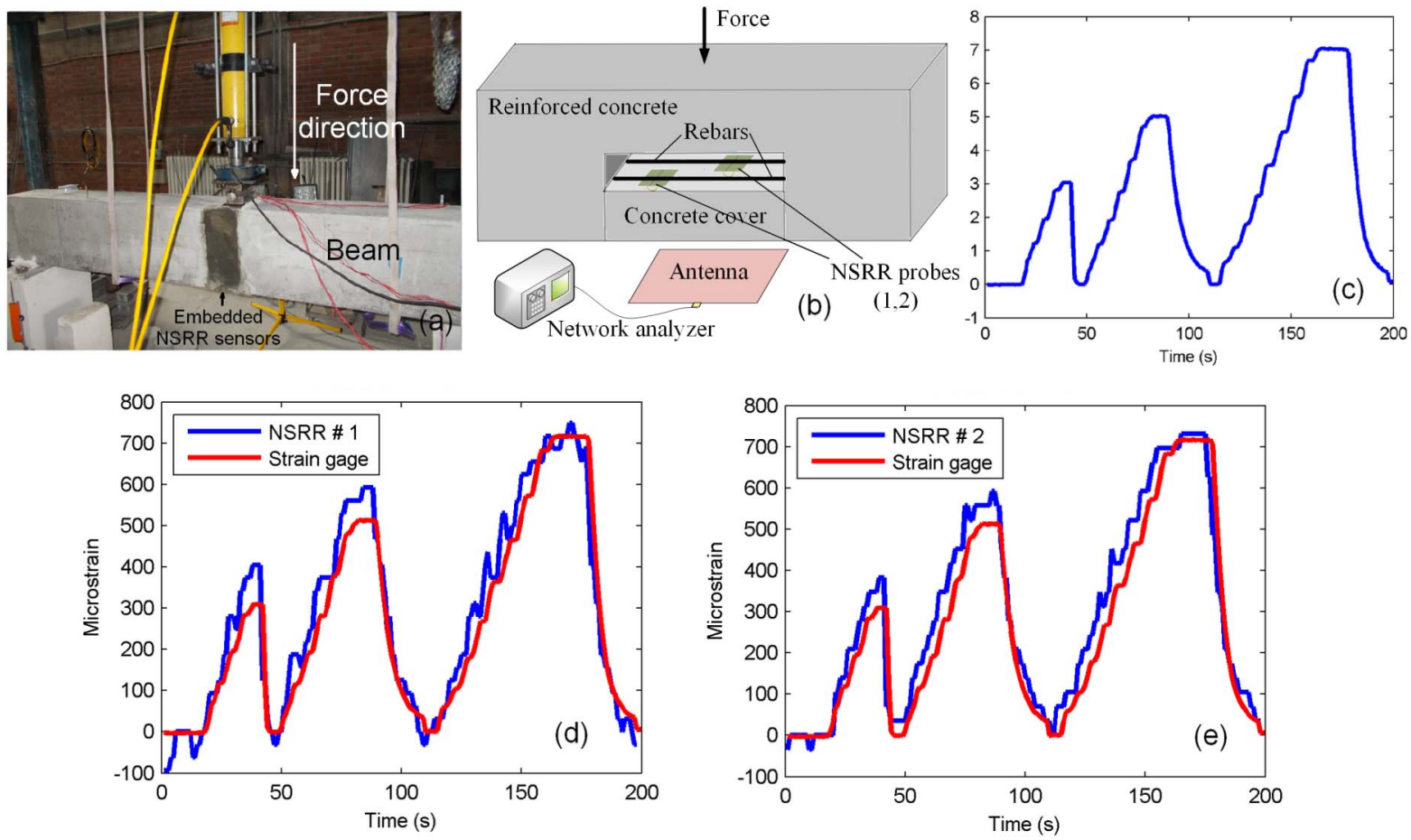

Fig. 9. Simultaneous multi-point sensing by two different sensors in a simply supported beam experiment: (a) Experiment setup. (b) Diagram showing the placement of NSRR probes on the reinforcing bars within the beam. (c) Applied force plotted versus time (load cell value). (d) NSRR \# 1 strain versus time compared with strain gage data. (e) NSRR \# 2 strain versus time compared with strain gage data.

other's resonance frequency. There are two options available to the designer: 1) To eliminate the inter-coupling as much as possible by keeping the array elements at a safe distance, e.g. a minimum distance of $20 \mathrm{~mm}$ can be selected for the comb-like NSRR case where the change of resonance frequency due to inter-coupling is as low as $0.1 \%, 2$ ) To keep the array elements at a distance where inter-coupling is still high, but to characterize the system accordingly, i.e. using the variation of resonance frequency with the sensed quantity under conditions where inter-coupling is dominant. Both are valid options since it is observed by the experiments that the sensitivity of sensing is not affected by inter-coupling.

\section{APPLICATION IN SHM}

An application of the multi-point sensing is in SHM, where the NSRR sensors can be integrated on reinforced concrete structural components such as beams or columns in order to measure the elongation or contraction on the reinforcing bars (rebars) embedded within these structures in time. When several of these sensors are placed at different locations inside a beam, it might be possible to obtain a readout from each of them at the same time by only an external antenna. Such quantitative information from different locations of a beam is very valuable for the assessment of the damage of the regarding component. In order to test the performance of the sensors in such a real-life scenario, an experiment was conducted and the results were published in a separate paper [25]. In this experiment, called the simply supported beam experiment, a vertical force was applied onto a reinforced concrete beam placed on two fulcrums at each end (see Fig. 9-a), leading to a horizontal elongation on the rebars present at the bottom of the beam. Two NSRR probes were placed on two different rebars located on the same rebar grid plane inside the beam (as depicted in Fig. 9-b). The dynamic strain measured by the sensors was compared with the results read from the wired strain gages attached on the same axial strain point in the surface of the beam, across the NSRR probe. Both our sensing system and the data acquisition system to which all the strain gages were connected simultaneously recorded data with a rate of $1 \mathrm{~s}$. In order to capture the frequency shift data with a high resolution, 1601 data points are used in the network analyzer, which corresponds to a sweeping time of around $1 \mathrm{~s}$. Therefore, the minimum time step that can be chosen is $1 \mathrm{~s}$, which is the value used in the experiments. As a result, the limit of the measurement rate is again dictated by the VNA, and other parameters such as the number of sensors do not have an effect on it. It should be noted that this strain buildup takes place over very large time periods for the application of structural health monitoring, e.g. months or years, hence a very short measurement time is not necessary. For the experiments, this natural process is shrunk to the minimum time possible, i.e. to the order of minutes. The applied force regime is shown in Fig. 9-c. The maximum force level during the loading was 7 tons, a value that stays within the elastic (linear) deformation region of steel where induced damage is reversible. The edge-to-edge distance between the NSRR probes was selected as $2 \mathrm{~cm}$ in order to minimize the effect of inter-coupling. Additionally, in order to undo the disruptive effect of the complex medium created by the rebars and the concrete on the coupling between 
the probes and the antenna, a 1-cm thick Styrofoam spacer was placed between the rebars and the probes, as suggested in [24]. The initial resonance frequencies of the two sensors were separated by changing their $d_{0}$ 's. The sensor strain data compared with strain gage data are all shown in Fig. 9. The frequency shift data of the NSRR probes were converted into displacement and then strain by a pre-experimental transformation curve obtained by the laboratory experiments. The matching of the NSRR \# 1 and NSRR \# 2 strain with the strain obtained via the strain gages shows that the wireless multi-point sensing concept is both functional and accurate in a real-life complex electromagnetic medium. As a proofof concept demonstration, only two NSRR probes are used. However, it is demonstrated by the controlled displacement experiments that the number of the NSRR probes can easily be increased. For this sensing scheme to be employed at a larger-scale structure, additional modifications must take place. Since it is not possible to track all the sensors in a building via a single antenna, the proper method is to take discrete-time measurements instead of the continuous monitoring, where the antenna (and the acquisition device such as the VNA) is brought in front of the respective beam or column in which multiple sensors have previously been integrated. The antenna can take measurements from all the sensors placed at this specific beam at the same time. These tests can be repeated periodically and the change of the state of each sensors can be monitored. For very critical points, a permanent system can be established by fixing the antenna just outside the beam and monitoring the beam continuously. For such a fixed system, the multiple-sensors single-antenna geometry is especially beneficial. However, for a practical application, instead of using a VNA, a new way of acquiring data which is more specific and application-oriented as well as cheaper should be considered.

\section{CONCLUSION}

In this study, a 2-D sensing scheme achieved by a singleantenna, multiple-NSRR-sensor geometry is presented. The proposed sensing scheme incorporates significant advantages due to the system being wireless and passive, as well as exhibiting a high sensitivity and resolution. Proof-of-concept experiments are carried out for the cases where the number of the elements in the array is 2, 3 and 6. However, in theory and as shown via simulations, the number of the elements can be increased to a lot more, only to be limited by the bandwidth and the illumination pattern of the antenna which determines the quality of coupling between the NSRR probes and the antenna. This is also demonstrated by a full-wave simulation in a $4 \times 4$ array. Differently from the previous works shown in literature, the sensing system can capture the information regarding to multiple probes wirelessly in a single frequency sweep. Each NSRR probe in the array can be tuned to a specific resonance frequency through modification of either $d_{0}$ or $l$. By characterizing the change of the resonance frequency versus a changing $d$, a calibration curve can be obtained for each sensor in the array. Besides the beneficial electromagnetic coupling between the NSRR probes and the antenna that makes the sensing system possible, a detrimental coupling is also present among the NSRR probes, which results in a deviation from this transformation curve by the additional capacitance that leads to a change in the resonance frequency. Results of the experiments conducted in this study prove that the effects of the inter-coupling can be prevented significantly by leaving a specific gap between the array elements. An important application example for the sensor is also demonstrated in this work, where a dual-probe system is embedded inside a concrete beam for monitoring the dynamic strain changes of the steel reinforcing bars. The results are shown to be closely matching with those of the strain gages, thus proving that the wireless multi-point sensing by NSRR probes can be used in SHM.

\section{REFERENCES}

[1] T. Chen, S. Li, and H. Sun, "Metamaterials application in sensing," Sensors, vol. 12, no. 3, pp. 2742-2765, 2012.

[2] Q. W. Ye, L. Y. Guo, M. H. Li, Y. Liu, B. X. Xiao, and H. L. Yang, "The magnetic toroidal dipole in steric metamaterial for permittivity sensor application," Phys. Scripta, vol. 88, no. 5, Oct. 2013, Art. no. 055002.

[3] A. I. Aristov, U. Zywietz, A. B. Evlyukhin, C. Reinhard, B. N. Chichkov, and A. V. Kabashin, "Laser-ablative engineering of phase singularities in plasmonic metamaterial arrays for biosensing applications," Appl. Phys. Lett., vol. 104, no. 7, Feb. 2014, Art. no. 071101.

[4] A. K. Horestani, J. Naqui, D. Abbott, C. Fumeaux, and F. Martín, "Twodimensional displacement and alignment sensor based on reflection coefficients of open microstrip lines loaded with split ring resonators," Electron. Lett., vol. 50, no. 8, pp. 620-622, Apr. 2014.

[5] A. Ebrahimi, W. Withayachumnankul, S. F. Al-Sarawi, and D. Abbott, "Dual-mode behavior of the complementary electric-LC resonators loaded on transmission line: Analysis and applications," J. Appl. Phys., vol. 116, no. 8, Aug. 2014, Art. no. 083705.

[6] H. Kairm et al., "Concept and model of a metamaterial-based passive wireless temperature sensor for harsh environment applications," IEEE Sensors J., vol. 15, no. 3, pp. 1445-1452, Mar. 2015.

[7] A. Ebrahimi, W. Withayachumnankul, S. F. Al-Sarawi, and D. Abbott, "Metamaterial-inspired rotation sensor with wide dynamic range," IEEE Sensors J., vol. 14, no. 8, pp. 2609-2614, Aug. 2014.

[8] J. Q. Wang et al., "Optical refractive nanosensor with planar resonators metamaterial," Opt. Commun., vol. 338, pp. 399-405, Mar. 2015.

[9] X. Wang, C. Luo, G. Hong, and X. Zhao, "Metamaterial optical refractive index sensor detected by the naked eye," Appl. Phys. Lett., vol. 102, no. 9, Mar. 2013, Art. no. 091902.

[10] M. Ren et al., "Isotropic spiral plasmonic metamaterial for sensing large refractive index change," Opt. Lett., vol. 38, no. 16, pp. 3133-3136, 2013.

[11] W. Withayachumnankul, K. Jaruwongrungse, C. Fumeaux, and D. Abbott, "Metamaterial-inspired multichannel thin-film sensor," IEEE Sensors J., vol. 12, no. 5, pp. 1455-1458, May 2012.

[12] M. Puentes, M. Maasch, and M. Schubler, and R. Jakoby, "Frequency multiplexed 2-dimensional sensor array based on split-ring resonators for organic tissue analysis," IEEE Trans. Microw. Theory Techn., vol. 60, no. 6, pp. 1720-1727, Jun. 2012.

[13] A. K. Horestani, J. Naqui, Z. Shaterian, D. Abbott, C. Fumeaux, and F. Martín, "Two-dimensional alignment and displacement sensor based on movable broadside-coupled split ring resonators," Sens. Actuators A, Phys., vol. 210, pp. 18-24, Apr. 2014.

[14] X. Xu and H. Huang, "Multiplexing passive wireless antenna sensors for multi-site crack detection and monitoring," Smart Mater. Struct., vol. 21, no. 1, p. 015004, Dec. 2012.

[15] R. Melik et al., "Nested metamaterials for wireless strain sensing," IEEE J. Sel. Topics Quantum Electron., vol. 16, no. 2, pp. 450-458, Mar./Apr. 2010.

[16] B. Ozbey et al., "Wireless displacement sensing enabled by metamaterial probes for remote structural health monitoring," Sensors, vol. 14, no. 1, pp. 1691-1704, Jan. 2014.

[17] B. Ozbey, H. V. Demir, O. Kurc, V. B. Erturk, and A. Altintas, "Wireless measurement of elastic and plastic deformation by a metamaterial-based sensor," Sensors, vol. 14, no. 10, pp. 19609-19621, Oct. 2014.

[18] R. Bhattacharyya, C. Floerkemeier, and S. Sarma, "Towards tag antenna based sensing - An RFID displacement sensor," in Proc. IEEE Int. Conf. RFID, Apr. 2009, pp. 95-102. 
[19] S.-D. Jang and J. Kim, "Passive wireless structural health monitoring sensor made with a flexible planar dipole antenna," Smart Mater. Struct., vol. 21 , no. 2, p. 027001 , Jan. 2012.

[20] S. Caizzone and G. Marrocco, "RFID-grids for deformation sensing," in Proc. IEEE Int. Conf. RFID, Apr. 2012, pp. 130-134.

[21] X. Yi, C. Cho, J. Cooper, Y. Wang, M. M. Tentzeris, and R. T. Leon, "Passive wireless antenna sensor for strain and crack sensing-Electromagnetic modeling, simulation, and testing," Smart Mater. Struct., vol. 22, no. 8, p. 085009, Jul. 2013.

[22] J. Kim, Z. Wang, and W. S. Kim, "Stretchable RFID for wireless strain sensing with silver nano ink," IEEE Sensors J., vol. 14, no. 12, pp. 4395-4401, Dec. 2014.

[23] C. Paggi, C. Occhiuzzi, and G. Marrocco, "Sub-millimeter displacement sensing by passive UHF RFID antennas," IEEE Trans. Antennas Propag., vol. 62, no. 2, pp. 905-912, Feb. 2014.

[24] B. Ozbey, H. V. Demir, O. Kurc, and V. B. Ertürk, and A. Altintas, "Wireless sensing in complex electromagnetic media: Construction materials and structural monitoring," IEEE Sensors J., vol. 15, no. 10 , pp. 5545-5554, Oct. 2015.

[25] B. Ozbey, V. B. Erturk, H. V. Demir, A. Altintas, and O. Kurc, "A wireless passive sensing system for displacement/strain measurement in reinforced concrete members," Sensors, vol. 16, no. 4, p. 496, Apr. 2016.

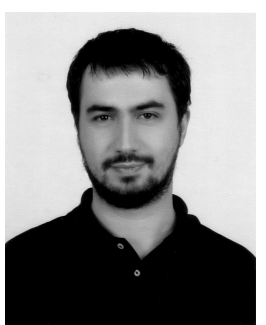

Burak Ozbey received the B.S., M.S., and $\mathrm{Ph} . \mathrm{D}$. degrees from Bilkent University, Ankara, Turkey, in 2008, 2011, and 2016, respectively, all in electrical and electronics engineering. His research interests include electromagnetic theory, microwave circuits, structural health monitoring, and design and testing of wireless RF sensors. He was a recipient of the 2016 Felsen Award for Excellence in Electromagnetics.

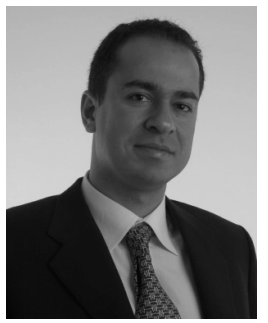

Vakur B. Ertürk (M'00) received the B.S. degree in electrical engineering from the Middle East Technical University, Ankara, Turkey, in 1993, and the M.S. and Ph.D. degrees from The Ohio State University, Columbus, in 1996 and 2000, respectively. He is currently an Associate Professor with the Electrical and Electronics Engineering Department, Bilkent University, Ankara. His research interests include the analysis and design of planar and conformal arrays, high-frequency techniques, structural health monitoring, magnetic resonance imaging, scattering from and propagation over large terrain profiles. He served as the Secretary/ Treasurer of the IEEE Turkey Section and the Turkey Chapter of the IEEE Antennas and Propagation, Microwave Theory and Techniques, Electron Devices, and Electromagnetic Compatibility Societies. He was a recipient of the 2005 URSI Young Scientist and the 2007 Turkish Academy of Sciences Distinguished Young Scientist Awards.

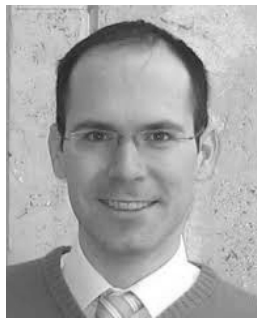

Ozgur Kurc received the B.S. and M.S. degrees in civil engineering from METU, Ankara, Turkey, and the Ph.D. degree in structural engineering from the Georgia Institute of Technology, Atlanta, GA, USA, in 2005. He is currently an Associate Professor of Structural Engineering with the Structural and Earthquake Engineering Laboratory, Department of Civil Engineering, METU. His current research interests involve wind and earthquake engineering, finite-element method, high-performance computing, and analysis and design of high-rise buildings.

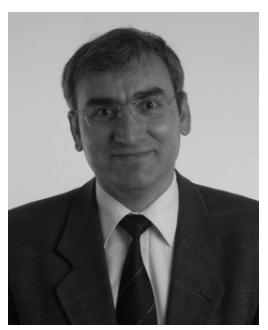

Ayhan Altintas (SM'93) received the B.S. and M.S. degrees from the Middle East Technical University, Ankara, Turkey, in 1979 and 1981, respectively, and the Ph.D. degree from The Ohio State University, Columbus, in 1986. From 1981 to 1987, he was with the ElectroScience Laboratory, Ohio State University. He is currently a Professor of Electrical Engineering with Bilkent University, Ankara. He has held Research Fellow and Guest Professor positions at Australian National University, Canberra, Australia, Tokyo Institute of Technology, Japan, Technical University of Munich, Germany, and Concordia University, Montréal, Canada. His Research interests include high-frequency and numerical techniques in electromagnetic scattering and diffraction, propagation modeling and simulation, and fiber and integrated optics. He is a member of Sigma Xi and Phi Kappa Phi. He is a Fulbright Scholar, and an Alexander von Humboldt Fellow. He is the President of URSI Turkish National Committee. He was a recipient of the IEEE Third Millennium Medal. He received the ElectroScience Laboratory Outstanding Dissertation Award of 1986, the IEEE 1991 Outstanding Student Branch Counselor Award, the 1991 Research Award of Prof. Mustafa N. Parlar Foundation of METU, and the Young Scientist Award of Scientific and Technical Research Council of Turkey in 1996. He was the Chairman of the IEEE Turkey Section from 1991 to 1993 and from 1995 to 1997.

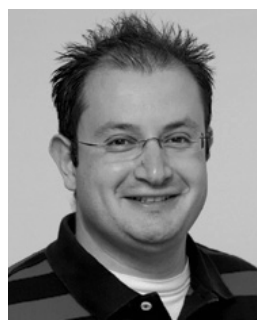

Hilmi Volkan Demir received the B.S. degree from Bilkent University in 1998 and the M.S and Ph.D. degrees in 2000 and 2004, respectively, from Stanford University, all in electrical engineering. He is named a Fellow by the Singapore National Research Foundation (NRF) and appointed to Full Professorship at Nanyang Technological University (NTU) Singapore. He is concurrently a Professor of Electrical Engineering and Physics at Bilkent University (his alma mater). His current research interests include the science of excitonics for high-efficiency light generation and harvesting, nanocrystal optoelectronics, implantable electronics and wireless sensing. He published over 200 peer-reviewed research articles in major scientific journals and delivered over 200 invited seminars, lectures and colloquia. He has contributed to commercialization and licensing of several new enabling technologies as well as establishing two successful companies. As a principal inventor, he generated over 30 patents, several of which have currently been used, owned or licensed by the industry. These scientific and entrepreneurship activities resulted in important international awards, including the Nanyang Award for Research Excellence, the European Science Foundation European Young Investigator Award, and The Outstanding Young Person in the World Award from the JCI Federation of Young Leaders and Entrepreneurs. 